\title{
Transient responses during hyperosmotic shock in the filamentous fungus Neurospora crassa
}

\author{
Correspondence \\ Roger R. Lew \\ planters@yorku.ca
}

Received 20 August 2008

Revised 13 October 2008

Accepted 16 October 2008

\author{
Roger R. Lew and Shanar Nasserifar \\ Department of Biology, York University, Toronto ON M3J 1P3, Canada
}

Fungal cells maintain an internal hydrostatic pressure (turgor) of about $400-500 \mathrm{kPa}$. In the filamentous fungus Neurospora crassa, the initial cellular responses to hyperosmotic treatment are loss of turgor, a decrease in relative hyphal volume per unit length (within $1 \mathrm{~min}$ ) and cell growth arrest; all recover over a period of 10-60 min due to increased net ion uptake and glycerol production. The electrical responses to hyperosmotic treatment are a transient depolarization of the potential (within $1 \mathrm{~min}$ ), followed by a sustained hyperpolarization (after $4 \mathrm{~min}$ ) to a potential more negative than the initial potential (a driving force for ion uptake). The nature of the transient depolarization was explored in the context of other transient responses to hyperosmotic shock, to determine whether activation of a specific ion permeability or some other rapid change in electrogenic transport was responsible. Changing the ionic composition of the extracellular medium revealed that $\mathrm{K}^{+}$permeability increases and $\mathrm{H}^{+}$permeability declines during the transient depolarization. We suggest that these changes are due to concerted inhibition of the electrogenic $\mathrm{H}^{+}$-ATPase, and an increase in a $\mathrm{K}^{+}$conductance. Knockout mutants of known $\mathrm{K}^{+}$ (tok, trk, trm-8, hak-1) and $\mathrm{Cl}^{-}$(a clc-3 homologue) channels and transporters had no effect on the transient depolarization, but trk and hak-1 do play a role in osmoadaptation, as does a homologue of a serine kinase regulator of $\mathrm{H}^{+}$-ATPase in yeast, Ptk2.

\section{INTRODUCTION}

Fungi often maintain a high internal hydrostatic pressure (turgor) during cellular expansion, a trait also common in walled cells of higher plants. In response to hyperosmotic conditions, the regulation of turgor relies upon osmolyte accumulation, both de novo synthesis of osmotically active metabolites (for example, glycerol, mannitol or erythritol) (Beever \& Laracy, 1986; Ellis et al., 1991) and ion uptake from the extracellular medium (Lew et al., 2006; Lew \& Levina, 2007). In Neurospora crassa, a mutant unable to accumulate glycerol (cut) can still accumulate ions and retains the ability to regulate turgor (Lew \& Levina, 2007). Thus, either mechanism is sufficient, offering redundant mechanisms in response to hyperosmotic shock. The transduction pathway that controls turgor regulation is the osmotic MAP kinase pathway. The pathway has been characterized most completely in yeast (O'Rourke et al., 2002), and is found in numerous fungal species on the basis of comparative genomics (Krantz et al., 2006). In N. crassa, a histidine kinase sensor OS-1 (Alex et al., 1996; Miller et al., 2002) and an associated response regulator RRG-1 (Jones et al., 2007) function upstream of the MAPKKK OS-4 and MAPKK OS-5 (Fujimura et al., 2003), and MAPK OS-2 (Zhang et al., 2002), to elicit both glycerol

Abbreviation: BTP, Bistris propane. accumulation (Noguchi et al., 2007) and ion accumulation (Lew et al., 2006) during turgor recovery after hyperosmotic shock.

One of the immediate responses to hyperosmotic shock in fungi is a transient depolarization of the electrical potential (Lew et al., 2004). In this paper, we explore the nature of this electrical response in the context of the time-courses of immediate (turgor loss, hyphal shrinkage and growth arrest) and long-term (sustained hyperpolarization and growth resumption during turgor recovery) responses to hyperosmotic shock.

\section{METHODS}

Strains. Stock cultures of wild-type (FGSC 2489) and knockout mutants (Colot et al., 2006) were obtained from the Fungal Genetics Stock Center (School of Biological Sciences, University of Missouri, Kansas City, Missouri, USA) (McCluskey, 2003). The following knockout mutants were used: tok (FGSC 12045), trk (FGSC 12678), trm-8 (FGSC 16162), hak-1 (FGSC 13816), a homologue of a chloride channel CLC-3 (FGSC 14929) and a homologue of yeast Ptk2 (FGSC 17932). To obtain a trk trm-8 double mutant, the trk ${ }^{-}$(FGSC 12678 mating type a) and $\mathrm{trm}-8^{-}$(FGSC16162 mating type A) strains were crossed using standard procedures (Davis \& de Serres, 1970). To identify the double mutant, DNA was extracted from the progeny and screened by PCR for sequences flanking the insertion site of the knockout cassette in $t r k^{-}$and $t r m-8^{-}$, and as an internal control, the wild-type gene sequences. A double trk trm-8 mutant was identified 
by the presence of the $t r k^{-}$and $t r m-8^{-}$PCR products and absence of the control (wild-type gene) products.

Stock cultures of the strains were maintained on Vogel's (plus $1.5 \%$, $\mathrm{w} / \mathrm{v}$, sucrose and $2.0 \%, \mathrm{w} / \mathrm{v}$, agar) medium (VM) (Vogel, 1956).

Culture preparation for experiments. Cultures were prepared by inoculating conidia onto strips $(2.5 \times 6 \mathrm{~cm})$ of dialysis tubing that overlay the VM in Petri dishes and incubated at $28{ }^{\circ} \mathrm{C}$ overnight. The dialysis tubing was cut with a razor blade to a size of about $1 \times 3 \mathrm{~cm}$, which included the growing edge of the colony, placed inside the cover of a $30 \mathrm{~mm}$ Petri dish, and immobilized on the bottom with masking tape. The culture was flooded with $3 \mathrm{ml}$ buffer solution (BS) (in $\mathrm{mM}$ ): $\mathrm{KCl}(10), \mathrm{CaCl}_{2}(1), \mathrm{MgCl}_{2}$ (1), sucrose (133) and MES (10), $\mathrm{pH}$ adjusted to 5.8 with $\mathrm{NaOH}$, or, for measurements of ion dependence, a modified BS. Dependent on the experimental run, modified BS contained (in $\mathrm{mM}$ ): $\mathrm{KCl}(0.1,1$ or 10$) \mathrm{CaCl}_{2}$ (0.1 or 10$)$, $\mathrm{MgCl}_{2}$ (1), sucrose (133) and MES (10); or with gluconate salts replacing the chloride salts $\left(0.1,1\right.$, or $10 \mathrm{~K}$-gluconate), $\mathrm{Ca}_{1 / 2^{-}}$ gluconate (2) and $\mathrm{Mg}_{1 / 2}$-gluconate (2); or was buffered with $10 \mathrm{mM}$ MES/BTP (Bistris propane) replacing $\mathrm{MES} / \mathrm{NaOH}(\mathrm{pH} 4,5,6$ or 7; the final $\mathrm{pH}$ was measured at the end of each experiment); or was buffered with sodium phosphate (10) instead of MES/NaOH. Growth of hyphae at the colony edge resumed within 15 min after flooding with the various BS modifications. In experiments comparing wildtype and the mutants, we normally interspersed measurements of each of the strains.

Electrical measurements. Large trunk hyphae $(10-20 \mu \mathrm{m}$ diameter) about $0.5 \mathrm{~cm}$ behind the colony edge were impaled with a micropipette. After a stable membrane potential was obtained, the hyphae were subjected to hyperosmotic conditions either by adding $0.5 \mathrm{ml} \mathrm{BS}$ (or modified BS) $+1 \mathrm{M}$ sucrose to the $3 \mathrm{ml}$ of BS (or modified BS) in the dish (a net increase of about 155 mosmol kg-1) in a circle around the objective, or by using perfusion with BS (or modified BS) plus $0.55 \mathrm{M}$ sucrose (unless otherwise specified), at a flow rate of $6.4 \mathrm{ml} \mathrm{min}{ }^{-1}$ (Econo Pump model EP-1, Bio-Rad), with inlet and outlet ports positioned close to and on opposite sides of the objective. Arrival of the osmoticum at the hypha could be confirmed by the refractive wave caused by the higher osmolarity of the treatment solution. Perfusion was used for most experiments (with the exception of some of the mutant screens) to assure the rapid and controlled arrival of osmoticum at the hypha. In either case, the rapid electrical responses occurred with similar timing and magnitude. The electrophysiological techniques have been described in detail elsewhere (Lew, 1996, 2007). Voltage clamping was performed using double-barrel micropipettes and a bipolar staircase of clamped voltages. Both the clamped voltage and clamping currents were measured during the last $5 \mathrm{~ms}$ of the $50 \mathrm{~ms}$ clamp. Experiments exhibiting voltage clamp fidelity were used for estimates of input conductances obtained by determining the slopes of the currentvoltage relations.

Hyphal volume per unit length measurements. To determine directly the relation between turgor and hyphal volume, and temporally correlate turgor loss with the transient depolarization, hyphal volumes were measured during hyperosmotic perfusion. Once growth had resumed at the colony edge, a trunk hypha about $0.5 \mathrm{~mm}$ behind the colony edge was imaged with a $\times 63$ water-immersion objective using an Orca C-4742-95 camera (Hammamatsu Photonics) and OpenLab 3.1.7 software (Improvision) during hyperosmotic perfusion. To ensure medial focusing of the hypha, it was sometimes necessary to stop the perfusion, focus, capture a digital image, and then restart perfusion. The diameter of the hypha was measured about $5 \mu \mathrm{m}$ above and below a septum (Fig. 1); the two measurements were averaged, then squared (to obtain a value proportional to hyphal volume per unit hyphal length). The relative

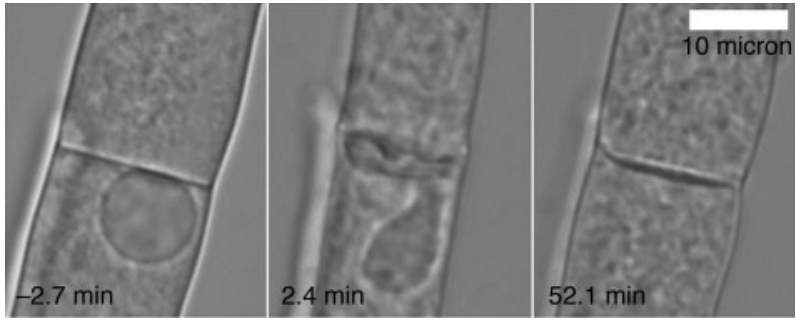

Fig. 1. Hyphal volume measurements. Images are shown before hyperosmotic treatment (BS) $(-2.7 \mathrm{~min})$, after the hypha had shrunk in response to perfusion with BS plus $0.55 \mathrm{M}$ sucrose (2.4 $\mathrm{min}$ ) and after turgor recovery (52.1 $\mathrm{min}$ ). Hyphal diameters about $5 \mu \mathrm{m}$ below and above the septa were measured, averaged, squared (to obtain relative hyphal volume per unit length) and normalized to the initial volume $\left(V / V_{i}\right)$ before hyperosmotic treatment.

hyphal volume per unit length was normalized to the initial volume $\left(V / V_{\mathrm{i}}\right)$.

Measurements of the intracellular hydrostatic pressure (turgor) were as described by Lew et al. (2004) and Lew \& Levina (2007). A micropipette filled with silicon oil was impaled into the cell, and the pressure required to bring the oil/cell sap interface to the micropipette tip was measured with a pressure transducer. Turgor is reported in $\mathrm{kPa}(1 \mathrm{bar}=100 \mathrm{kPa}, 1 \mathrm{~atm}=101.3 \mathrm{kPa})$.

Respiration measurements. Germlings were used for $\mathrm{O}_{2}$ consumption measurements to ensure adequate mixing of the cell suspension. Conidia were harvested by release in a small volume of sterile distilled $\mathrm{H}_{2} \mathrm{O}$, filtered through cheesecloth, inoculated into $125 \mathrm{ml}$ Erlenmeyer flasks containing $25 \mathrm{ml} \mathrm{VM}$ plus $2 \%$ sucrose and incubated in a reciprocating shaker $(100$ r.p.m. $)$ at $28{ }^{\circ} \mathrm{C}$. Germination and hyphal growth were monitored by microscopy. The percentage germination was near $100 \%$, with hyphal extension lengths of a few to $100 \mu \mathrm{m}$ after about $5 \mathrm{~h}$. The germling suspension was aerated by vigorous shaking and $2 \mathrm{ml}$ aliquots placed in the oxygen electrode chamber (Hansatech model DW1AD). $\mathrm{O}_{2}$ consumption rates were measured for 60-100 s. Then, osmoticum (either $\mathrm{KCl}$ from a $3 \mathrm{M}$ stock, or sucrose from a $2 \mathrm{M}$ stock) was added, and $\mathrm{O}_{2}$ consumption rates were measured for 60-100 s (the time frame of the transient depolarization). To reach a suitable final concentration of osmoticum, a significant volume of osmoticum had to be added (up to $0.75 \mathrm{ml}$ ). Therefore, for experimental controls, VM was added at the same volume in interspersed measurements. Data are presented as a percentage of the initial $\mathrm{O}_{2}$ consumption rate.

Statistical analysis. Data are shown as mean \pm SD (sample size) unless stated otherwise. Independent two-tail $t$-tests and nonlinear regressions were performed in Excel (Microsoft), SYSTAT (Systat, Inc.), or KaleidaGraph (Synergy Software).

\section{RESULTS}

To understand the role of the transient potential change in turgor regulation, we initially determined its timing during the multiple changes that occur after the imposition of a hyperosmotic treatment. 


\section{Time-course of hyperosmotic-induced hyphal changes}

The changes known to occur immediately after hyperosmotic treatment, and during turgor recovery, are shown in Fig. 2. The electrical changes are an immediate transient depolarization followed by a sustained hyperpolarization of the potential. The maximal depolarization occurred $0.96 \pm 0.36 \mathrm{~min}(n=77)$ after commencement of osmotic perfusion. The subsequent hyperpolarization, measured as the time for the potential to become more negative than the initial potential, was $4.13 \pm 2.36 \min (n=45)$ after commencement of the hyperosmotic treatment. The rapid drop in turgor was followed by a recovery to near original

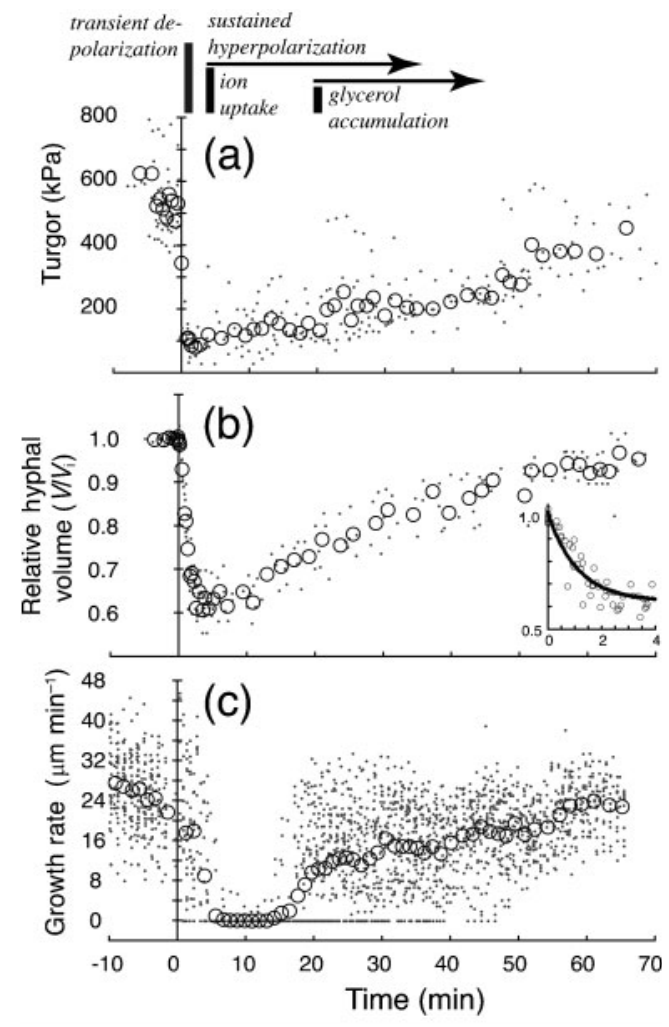

Fig. 2. Time-courses of responses to hyperosmotic treatment. The average time to peak depolarization and onset of sustained hyperpolarization are indicated by vertical bars, as are the onset of ion uptake (Lew et al., 2006, 2008) and glycerol production (Lew \& Levina, 2007). Turgor (a) (the turgor data were previously published in Lew et al., 2004; Lew \& Levina, 2007) declines rapidly, as does relative hyphal volume per unit length $\left(V / V_{i}\right.$, see Fig. 1) (b). The inset (relative hyphal volume, $V I V_{i}$, vs time, min) shows the rapid exponential decline in volume. Hyphal tip growth rates (c) were measured at the edges of colonies grown between two layers of Cellophane (to avoid tip growth in the $z$-direction), retarding the arrival of the hyperosmotic treatment at the hyphae, so that inhibition of hyphal growth appears to lag behind the declines in turgor and hyphal volume. Individual data (small points) and means (circles) ( $n=4$ and 5 for hyphal volume and turgor, respectively, and 62 for growth rate measurements) are shown. values within 60 min (Fig. 2a) (Lew et al., 2004, 2008). Turgor loss occurred in concert with a decline in relative hyphal volume per unit length (Fig. 2b). The decline in relative hyphal volume per unit length immediately after hyperosmotic perfusion was exponential, with a time constant $( \pm \mathrm{SE})$ of $1.11 \pm 0.11 \mathrm{~min}$ (Fig. 2b, inset). Hyphal tip extension was measured at the edge of colonies grown between two Cellophane sheets (to avoid growth in the $z$-direction), so there was a lag in growth inhibition due to slowed arrival of the hyperosmotic treatment. Subsequently, inhibition of hyphal tip extension occurred within a similar time frame (Fig. 2c), and then recovered in concert with turgor and hyphal volume recovery as a consequence of ion uptake (commencing after $5 \mathrm{~min}$ ) and glycerol production (after $20 \mathrm{~min}$ ) (Lew et al., 2006; Lew \& Levina, 2007).

The relations between the three associated parameters turgor, volume and growth rate - are shown in Fig. 3. Temporal correlations between turgor, relative hyphal
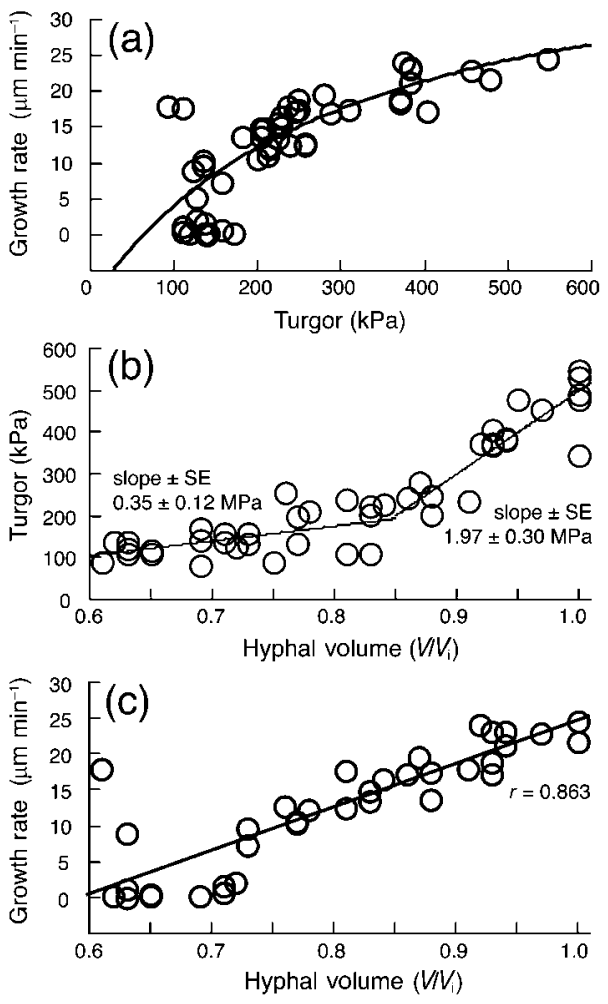

Fig. 3. Relations between hyphal growth rate, turgor and relative hyphal volume per unit length. The time-course data in Fig. 2 were aligned (within 1-2 $\mathrm{min}$ ) to match the three measured parameters. (a) Best fit of a Michaelis-Menten function (solid line) for growth rate vs turgor. (b) The linear slopes of turgor vs relative hyphal volume per unit length $\left(V / V_{\mathrm{i}}\right)$ are an estimate of the modulus of elasticity $( \pm S E)$ for the cell wall. (c) Growth rate exhibits a linear dependence on hyphal volume. Data with high growth rates at low turgor (a) and hyphal volume (c) are due to the lag in growth cessation after hyperosmotic treatment (Fig. 2c). 
volume per unit length and growth rate (Fig. 2) indicate dependence between growth and either turgor (Fig. 3a) or hyphal volume (Fig. 3b). The dependence of growth rate on turgor was fitted to a Michaelis-Menten function; a minimal turgor (about $100 \mathrm{kPa}$ ) may be required for hyphal growth under our experimental conditions [discounting the data with high growth rates due to the lag in growth cessation after hyperosmotic treatment (Fig. 2c)]. The relation between relative hyphal volume per unit length and turgor was nonlinear, expected if the modulus of elasticity $(\varepsilon)$ is a function of turgor (Husken et al., 1978). The modulus of elasticity describes the dependence of cell volume on pressure: $\Delta P=\varepsilon \cdot\left(\Delta V / V_{\mathrm{i}}\right)$ (Philip, 1958; Husken et al., 1978). In the case of the relation between turgor and relative hyphal volume per unit length (Fig. 3b) for $N$. crassa hyphae, there was a biphasic response. At low turgor $(80-200 \mathrm{kPa})$, the modulus of elasticity was $0.35 \pm 0.12 \mathrm{MPa}$ (slope $\pm \mathrm{SE}$ ), while at higher turgor (250$550 \mathrm{kPa}$ ), the change in volume was less, resulting in a higher modulus of elasticity $(1.97 \pm 0.30 \mathrm{MPa})$ (similar to the $1.36 \mathrm{MPa}$ modulus measured with a pressure probe; Lew et al., 2004). There was a linear relation between growth rate and relative hyphal volume per unit length (Fig. 3c) $[r=0.863$ when data with high growth rates due to the lag in growth cessation after hyperosmotic treatment (Fig. 2c) were included in the linear regression].

\section{Dual electrical responses to high osmolarity}

Treatment with high osmolarity caused two distinct electrical responses. At low or high osmolarity $(0.25$ or $0.55 \mathrm{M}$ sucrose), there was a sustained hyperpolarization that commenced about 4-5 min after the onset of sucrose treatment (Fig. 4, middle and lower panels). At high osmolarity, the sustained hyperpolarization was preceded by a transient depolarization (Fig. 4, lower panel). These dual responses were also observed when $\mathrm{KCl}$ was used as the osmoticum. At a concentration of $0.125 \mathrm{M}$, the initial potential of $-156 \pm 12$ depolarized only slightly (to $-141 \pm 11 \mathrm{mV}$ ) followed by a sustained hyperpolarization to $-183 \pm 14 \mathrm{mV}(n=5)$. At high $\mathrm{KCl}(0.3 \mathrm{M})$, the initial potential of $-150 \pm 5$ depolarized to $-23 \pm 14 \quad(n=5)$, followed by a sustained hyperpolarization to $-172 \pm 4 \mathrm{mV}$ $(n=3)$. Thus, the dual electrical responses are unrelated to the nature of the osmoticum, either sugar (sucrose) or salt $(\mathrm{KCl})$.

The concentration dependence of the transient depolarization (Fig. 5) was examined in detail. At sucrose concentrations below about $0.4 \mathrm{M}$, the transient depolarization was slight, if present at all. The magnitude of the transient depolarization was about $+100 \mathrm{mV}$ at sucrose concentrations of $0.55 \mathrm{M}$ or above (Fig. 4). Thus, a sucrose treatment of $0.55 \mathrm{M}$ was used to explore the ionic cause of the transient depolarization.

\section{Ionic effects on the transient depolarization}

To identify the ionic conductances responsible for the transient depolarization, the mycelium was bathed in

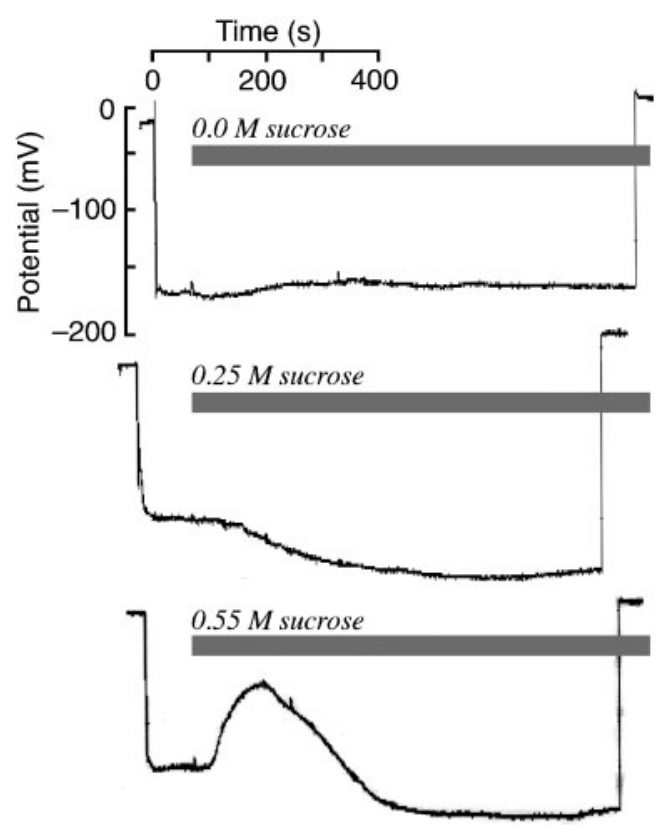

Fig. 4. Dependence of electrical changes on sucrose concentration: examples of electrical responses to treatments of $0,0.25$ and $0.55 \mathrm{M}$ sucrose (in BS) (as marked). At low sucrose (0.25 M), the transient depolarization is absent, but the sustained hyperpolarization is observed. Higher sucrose $(0.55 \mathrm{M})$ causes both the transient depolarization and sustained hyperpolarization.

buffer solutions (BS) modified by adjusting $\left[\mathrm{K}^{+}\right],\left[\mathrm{Cl}^{-}\right]$(by gluconate salt subsititutions) and $\mathrm{pH}$ (using BTP/MES). The basic premise was to change the Nernst potential $\left(E_{\mathrm{N}}\right)$ for individual ions by changing the extracellular ion concentration: $E_{\mathrm{N}}=(\boldsymbol{R T} / z F) \cdot \ln \left(\left[N_{\mathrm{o}}\right] /\left[N_{\mathrm{i}}\right]\right)$, where $\boldsymbol{R}$ is the gas constant $\left(8.314 \mathrm{~J} \mathrm{~mol}^{-1} \mathrm{~K}^{-1}\right), T$ is the temperature (room temperature ranged from 21 to $25{ }^{\circ} \mathrm{C}$; the average was $23{ }^{\circ} \mathrm{C}$, or $296.15 \mathrm{~K}$ ), $z$ is the ion valence, $F$ is the

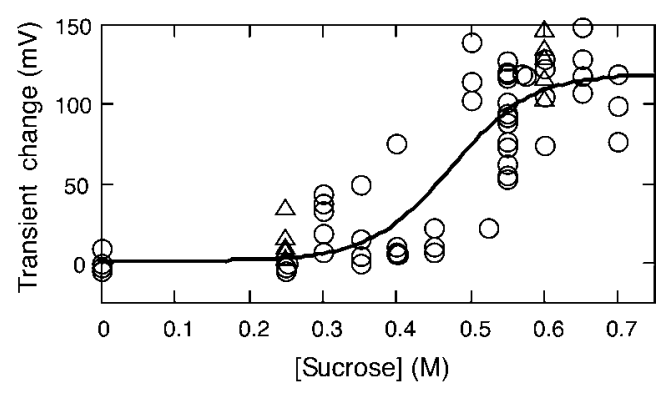

Fig. 5. Dependence of the transient depolarization on osmoticum concentration. The magnitude of the transient change in the potential (initial potential minus the peak transient depolarized potential) (circles) is shown as a function of [sucrose]. Data for $\mathrm{KCl}$ (at double the actual concentration, to account for the osmotically active $\mathrm{K}^{+}$and $\mathrm{Cl}^{-}$) are also included (triangles). The best fit is to a sigmoid function. 
Faraday constant $\left(9.649 \mathrm{~J} \mathrm{~mol}^{-1} \mathrm{~V}^{-1}\right)$, and $\left[N_{\mathrm{o}}\right] /\left[N_{\mathrm{i}}\right]$ is the ratio of ion concentrations outside and inside the hypha. The predicted change for a 10-fold change in extracellular ion concentration was $58.7 \mathrm{mV}$ for monovalent ions, and $29.2 \mathrm{mV}$ for divalent ions. These expected changes were compared to the actual changes. After hyphal growth resumed following immersion in the modified BS, impalement and stabilization of the potential, the hypha was perfused with the hyperosmotic treatment (modified BS plus $0.55 \mathrm{M}$ sucrose).

The effects of $\mathrm{KCl}$ and $\mathrm{pH}$ on the initial potential, the depolarized potential and the final sustained hyperpolarized potential are shown in Fig. $6 .\left[\mathrm{K}^{+}\right]$and $\left[\mathrm{H}^{+}\right]$ dependencies were determined from linear regressions using least-squares best fits and are reported as slope \pm SE. The initial potential exhibited some dependence (13.8 $\mathrm{mV} \pm 2.7$ per 10 -fold increase in $\left[\mathrm{K}^{+}\right], 15.2 \pm 1.4 \mathrm{mV}$ per 10 -fold increase in $\left.\left[\mathrm{H}^{+}\right]\right)$. The transient depolarization was affected more by $\left[\mathrm{K}^{+}\right](32.2 \pm 7.5 \mathrm{mV}$ per 10 -fold increase in $\left.\left[\mathrm{K}^{+}\right]\right)$, than by $\left[\mathrm{H}^{+}\right](9.4 \pm 3.4 \mathrm{mV}$ per 10 -fold increase in $\left.\left[\mathrm{H}^{+}\right]\right)$. Thus, the transient depolarization was associated with an increased permeability to $\mathrm{K}^{+}$, but not to the extent that the change in potential could be ascribed solely to $\mathrm{K}^{+}$ (in which case the expected dependence would have been $58.7 \mathrm{mV}$ per 10 -fold increase in $\left.\left[\mathrm{K}^{+}\right]\right)$. Conductance was measured before and during the transient depolarization for $[\mathrm{KCl}]$ and [K-gluconate] experiments. The initial conductances ( 274 to $566 \mathrm{nS}$ ) declined in $7 / 8$ experiments during the transient depolarization (by $126 \pm 88 \mathrm{nS}, n=9$ ) (data not shown; see also Lew et al., 2006; Lew \& Levina, 2007). The sustained hyperpolarization exhibited dependences similar to the initial potential $(6.2 \pm 5.7 \mathrm{mV}$ per 10 fold change in $\left[\mathrm{K}^{+}\right]$and $14.9 \pm 4.3 \mathrm{mV}$ per 10 -fold change in $\left.\left[\mathrm{H}^{+}\right]\right)$. An effect for $\mathrm{Cl}^{-}$can be discounted since a $\mathrm{Cl}^{-}$ conductance would have resulted in a more negative transient potential with increasing $\left[\mathrm{Cl}^{-}\right]$, rather than the observed positive trend. Experimental runs using 0.1, 1.0 and $10.0 \mathrm{mM} \mathrm{K}$-gluconate (to remove all $\mathrm{Cl}^{-}$from the extracellular medium) exhibited a negative change of $-17.5 \pm 7.1 \mathrm{mV}$ per 10 -fold increase in $\left[\mathrm{K}^{+}\right]$for the transient depolarization. This was unexpected for activation of a $\mathrm{K}^{+}$conductance, and cannot be explained by a $\mathrm{Cl}^{-}$conductance (in which case, no effect on the transient depolarization would have been expected since $\left[\mathrm{Cl}^{-}\right]$was unchanged). Gluconate influx through an anion conductance could explain the negative trend. The one other 'natural' anion that was examined as a possible contributor was phosphate: elevating extracellular phosphate to $10 \mathrm{mM}$ (as sodium phosphate, $\mathrm{pH}$ 5.8) would be expected to have a large effect on the transient depolarization if an anion (phosphate) conductance played a role, but the transient depolarization $(-64 \pm 44 \mathrm{mV}, n=6)$ was similar to that seen with standard BS $(-51 \pm 26 \mathrm{mV}, n=21)$, which contains no phosphate.

$\mathrm{Ca}^{2+}$ channels do not play a role in the hyperosmotic shock. The initial potential and the depolarized potential exhibited no significant dependence on $\left[\mathrm{Ca}^{2+}\right](2.8 \pm 2.5$ $\mathrm{mV}$ and $5.5 \pm 7.2 \mathrm{mV}$ per 10 -fold change in $\left[\mathrm{Ca}^{2+}\right]$, respectively). Instead, the increase in $\left[\mathrm{K}^{+}\right]$dependence after hyperosmotic treatment suggested that an activated $\mathrm{K}^{+}$ conductance contributes to the transient depolarization, but other electrogenic transport must also be affected.

\section{Mutant screens for transient depolarization}

To determine whether the presence of a transient depolarization could be attributed to a specific transporter or transport regulator, knockout mutants were screened for the presence of the hyperosmotic-induced transient depolarization (and sustained final hyperpolarization) (Table 1). TOK was identified as an outward-rectifying $\mathrm{K}^{+}$channel by Roberts (2003); the tok mutant did exhibit a

(a)

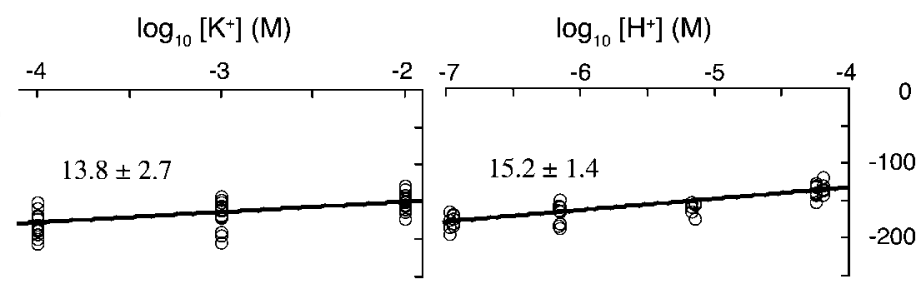

(b)

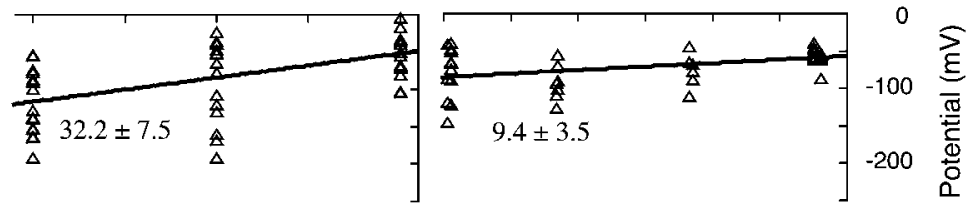

(c)

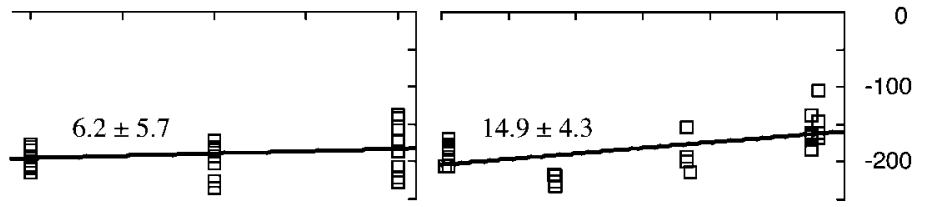

Fig. 6. Dependence of potential on extracellular $\left[\mathrm{K}^{+}\right]$and $\left[\mathrm{H}^{+}\right]$. The dependencies are shown as slopes $\left.\left(\mathrm{mV} / \log _{10}\left[\mathrm{M}^{+}\right]\right) \pm \mathrm{SE}\right)$ for the initial potential (a), the transient depolarization (b) and sustained hyperpolarization (c), for $\mathrm{K}^{+}$ (left panels) and $\mathrm{H}^{+}$(right panels). 
Table 1. Electrical responses of transport mutants to hyperosmotic treatment

The mean $\pm \mathrm{SD}(n)$ and $t$-test comparisons with wild-type are shown for knockout mutants of $\mathrm{K}^{+}$(tok, trk, trm-8, hak-1) and $\mathrm{Cl}^{-}(\mathrm{clc}-3 \mathrm{homologue})$ transport, and a putative $\mathrm{H}^{+}$-ATPase regulator (Ptk2 homologue). $E_{\mathrm{m}}$, membrane potential.

\begin{tabular}{|llll|}
\hline Mutant (FGSC no., NCU locus no.) & \multicolumn{1}{c|}{ Initial $\boldsymbol{E}_{\mathbf{m}}$} & Transient depolarization & Final $\boldsymbol{E}_{\mathbf{m}}$ \\
\hline Wild-type (2489) & $-155 \pm 13(22)$ & $-51 \pm 26(22)$ & $-178 \pm 31(10)$ \\
clc-3 (14929, NCU06624) & $-152 \pm 12(29) P=0.342$ & $-75 \pm 38(8) P=0.140$ & $-185 \pm 32(9) P=0.705$ \\
tok (12045, NCU04065) & $-164 \pm 18(15) P=0.103$ & $-73 \pm 43(15) P=0.093$ & $-176 \pm 36(11) P=0.850$ \\
trk (12678, NCU06449) & $-195 \pm 13(9) P<0.001$ & $-37 \pm 17(9) P=0.078$ & $-248 \pm 24(2)$ \\
trm-8 (16162, NCU02456) & $-157 \pm 10(4) P=0.797$ & $-36 \pm 7(4) P=0.028$ & $-187 \pm 10(4) P=0.468$ \\
trk trm-8 (12678X16162) & $-166 \pm 16(11) P=0.061$ & $-46 \pm 30(10) P=0.604$ & $-184 \pm 37(9) P=0.750$ \\
hak-1 (13816, NCU00790) & $-168 \pm 14(7) P=0.061$ & $-78 \pm 32(7) P=0.084$ & $-213 \pm 17(4) P=0.026$ \\
Ptk2 homologue (17932, NCU01940) & $-118 \pm 20(13) P<10^{-5}$ & $-64 \pm 37(13) P=0.291$ & $-128 \pm 33(12) P=0.002$ \\
& & & \\
\hline
\end{tabular}

more negative depolarization than wild-type, suggesting that the channel might normally play a role, but the difference was not statistically significant. The $\mathrm{K}^{+}$uptake transporter mutants of trk (Haro et al., 1999), trm-8 (encoding a homologue of the yeast TRK1; Kiranmayi \& Mohan, 2006) and the double mutant trk trm-8 all exhibited transient depolarizations that were more positive than wild-type. More positive depolarizations may reflect lower $\mathrm{K}^{+}$accumulation in the potassium transporter knockout mutants during growth in VM prior to the experiment, resulting in a more positive Nernst potential for $\mathrm{K}^{+}$compared to wild-type. The high-affinity potassium transporter knockout hak-1 (Haro et al., 1999) exhibited a more negative transient depolarization, as did the chloride channel knockout clc-3 (NCU06624, encoding a homologue of the mammalian CLC-3 based on BLAST comparisons).

Both the initial potential and sustained hyperpolarization were significantly depolarized compared to wild-type in a knockout mutant of a homologue (based on BLAST comparisons) of a kinase regulator of the $\mathrm{H}^{+}$-ATPase (Ptk2; NCU01940) in yeast (Eraso et al., 2006).

Long-term survival of the transport mutants in hyperosmotic conditions was also examined to assess their role in turgor recovery. Colony growth on agar of the $c l c-3$, tok and trm-8 mutants was insensitive to hyperosmotic conditions (VM plus $1.5 \%$ glucose and $1.2 \mathrm{M} \mathrm{NaCl}$ ). The trk and trk trm- 8 mutants exhibited some inhibition (about $50 \%$ compared to wild-type), as did the hak-1 mutant (about $80 \%$ ) and the Ptk2 homologue (about $30 \%$ ), but they were less sensitive to hyperosmotic conditions than osmosensitive mutants such as cut, os-1 and $o$ - 2 , which do not grow at $0.68 \mathrm{M} \mathrm{NaCl}(4 \%$, w/v) (Lew et al. 2006; Lew \& Levina, 2007).

In general, none of the knockout mutants examined had an unequivocal effect on the transient depolarization; therefore, none can be solely responsible or obligatory for the transient response. It is likely that more than one transporter is responsible, including transient shutdown of the plasma membrane $\mathrm{H}^{+}$pump.

\section{The $\mathrm{H}^{+}$pump may play a role in the transient depolarization}

The magnitude of the transient depolarization is similar to the depolarization that occurs when the $\mathrm{H}^{+}$pump is inhibited by ATP depletion using metabolic inhibitors. Using $5 \mathrm{mM}$ sodium cyanide, we observed a depolarization to $-47 \pm 20(n=13) \mathrm{mV}$ (Lew et al., 2008), similar to the maximal depolarization observed after hyperosmotic treatment with $0.55 \mathrm{M}$ sucrose $(-60 \pm 27 \mathrm{mV}, n=13)$. To test whether the transient depolarization was caused solely by hyperosmotic inhibition of the pump, the hypha was pre-treated with $5 \mathrm{mM}$ sodium cyanide to inhibit the pump, then with BS plus $0.55 \mathrm{M}$ sucrose in the continued presence of cyanide. Even after depolarization with cyanide, hyperosmotic treatment caused an additional depolarization, albeit small in magnitude (Fig. 7). From an initial potential of $-164 \pm 12 \mathrm{mV}$, cyanide depolarized the potential to $-57 \pm 15$; the hyperosmotic treatment caused an additional depolarization to $-33 \pm 8 \mathrm{mV}(n=12)$. A similar response was observed for the tok mutant: the initial potential of $-174 \pm 12 \mathrm{mV}$ was depolarized to $-68 \pm 21 \mathrm{mV}$ by cyanide $(n=9)$, and further depolarized to $-41 \pm 12 \mathrm{mV}(n=7)$ by hyperosmotic treatment.

\section{Respiration is unaffected by hyperosmotic treatment}

If the transient depolarization is caused in part by inhibition of the $\mathrm{H}^{+}$pump, it might lower ATP turnover due to a realignment of energy demands by the cell. Respiratory activity was therefore examined to determine whether hyperosmotic treatment caused a decrease in ATP turnover within 2-3 min of treatment (the time frame of the transient depolarization). Rates of respiration were normalized as percentages of the initial rate. The control (treatment with VM) respiratory rate declined to $80 \pm 13 \%$ of initial $(n=13)$ (due to dilution of the germling suspension). Treatment with sucrose (final concentration $545 \mathrm{mM}$ ) and $\mathrm{KCl}$ (final concentration $390 \mathrm{mM}$ ) caused similar declines: $83 \pm 13 \%(n=7)$ and $83 \pm 6 \% \quad(n=5)$, respectively. Thus, there is no change in respiration immediately after hyperosmotic treatment. 

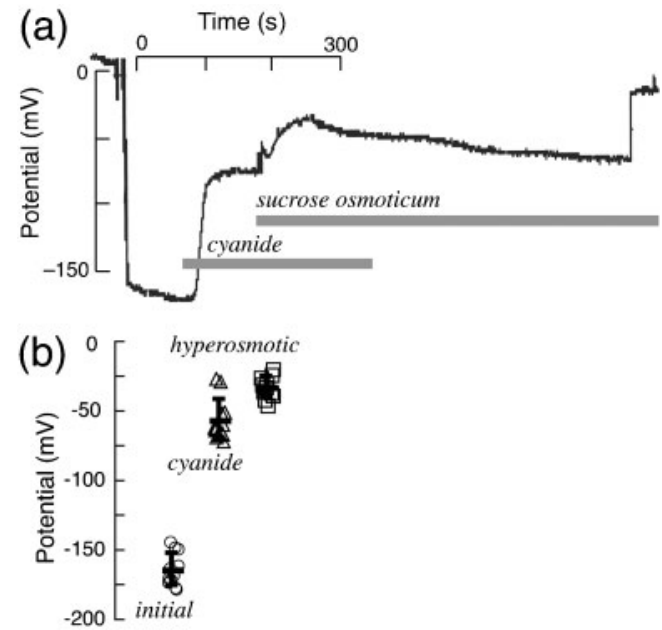

Fig. 7. Electrical response to hyperosmotic shock after cyanide inhibition of the $\mathrm{H}^{+}$pump. (a) An example of an experimental trace in which hyperosmotic treatment caused a depolarization after cyanide treatment, indicating that $\mathrm{H}^{+}$pump activity is not the sole contributor to the hyperosmotic-induced transient depolarization. Subsequent washout of cyanide did not result in recovery of the potential, indicative of substantial damage caused by the dual cyanide and hyperosmotic treatments. (b) Compiled data for the initial potential (circles), depolarized potential after cyanide treatment (triangles) and potential after hyperosmotic treatment in the continued presence of cyanide (squares). Similar results were obtained with the tok mutant (not shown).

\section{DISCUSSION}

The high internal hydrostatic pressure (turgor) of walled cells is a consequence of the high osmolarity of the intracellular cytoplasm compared to the external osmolarity. Many walled cells maintain their turgor by the biosynthesis of osmotically active metabolites (Jennings, 1995; Bohnert \& Jensen, 1996) and ion uptake (Lew et al., 2006). Turgor is believed to drive cellular expansion, and intrahyphal pressure gradients may move cytoplasm forward with the growing tip (Lew, 2005), but not all hyphal organisms regulate turgor (Kaminskyj et al., 1992; Lew et al., 2004), and there are examples of amoeboid fungal cells (the slime mutant of $N$. crassa) and oomycetes (Money \& Harold, 1993) that grow in the absence of turgor. Thus, tip-growing organisms can access alternative pathways to growth and morphogenesis, allowing longterm survival. Nevertheless, a causal relation between turgor and cell expansion in walled cells is widely accepted (Bartnicki-Garcia et al., 2000). Temporal correlations between turgor, cell growth and relative hyphal volume per unit length (Figs 2 and 3) indicate that cellular expansion is fundamentally linked with increases in hyphal volume, mediated by turgor. The poor turgor regulation and growth of $N$. crassa os mutants (notably os- 1 and os-2) at high osmolarity (Lew et al., 2006) also links growth with turgor.
When subjected to hyperosmotic shock, fungal hyphae respond rapidly. Initially, turgor loss, hyphal shrinkage, growth arrest and electrical changes occur, followed by ion uptake from the extracellular solution and biosynthesis of osmolytes, such as glycerol. In N. crassa, the osmotic MAP kinase cascade regulates both glycerol production and ion uptake - activating the $\mathrm{H}^{+}$-ATPase to mediate an increase in net ion influx - resulting in turgor recovery within $60 \mathrm{~min}$ of hyperosmotic stress (Lew et al., 2006).

The fastest responses are the transient depolarization of the potential and hyphal shrinkage. The time to peak depolarization $(0.96 \pm 0.36 \mathrm{~min})$ is very similar to the time constant for hyphal shrinkage $(1.11 \pm 0.11 \mathrm{~min})$. Whether hyphal shrinkage causes the transient depolarization, via some sensing mechanism, is not clear. One candidate for sensing membrane deformation caused by hyphal shrinkage (and causing the transient depolarization) is the mechanosensitive $\mathrm{Ca}^{2+}$ channel, MID-1, but a mid-1 mutant exhibits a transient depolarization similar to wildtype (Lew et al., 2008). To gain insight into the nature of the transient depolarization, we varied the extracellular ion composition to determine which ion permeabilities change in response to hyperosmotic shock.

The dependence of the electrical potential on external cations was characterized in detail by Slayman (1965), who reported significant permeabilities to both $\mathrm{K}^{+}$and $\mathrm{H}^{+}$. We examined the changes caused by hyperosmotic treatment: the $\left[\mathrm{K}^{+}\right]$dependence increased markedly while $\left[\mathrm{H}^{+}\right]$ dependence declined. Thus, an increase in $\mathrm{K}^{+}$permeability occurs concomitant with a decline in $\mathrm{H}^{+}$permeability. We have not been able to identify the cause of the increased $\mathrm{K}^{+}$ permeability, based on mutant screens of likely candidates. Since the conductance declined irrespective of the extracellular $\left[\mathrm{K}^{+}\right]$, a $\mathrm{K}^{+}$conductance is unlikely to be the sole cause of the transient depolarization. The decline in $\mathrm{H}^{+}$ dependence can be attributed to a decrease in $\mathrm{H}^{+}$pump activity and concordant electrogenic $\mathrm{H}^{+}$/symport transport. Because the hyperosmotic-induced transient depolarization is similar to the depolarization caused by pump inhibition due to ATP depletion, and conductance is unchanged (Lew et al., 2006; Lew \& Levina, 2007) or declines, transient inhibition of the $\mathrm{H}^{+}$pump is likely to be a major contributor to the transient depolarization. ATP consumption by the pump is estimated to consume about $30 \%$ of ATP turnover (Slayman et al., 1973). Since we did not observe a hyperosmotic-induced change in $\mathrm{O}_{2}$ consumption, we suggest that ATP normally utilized by the pump is redirected towards other metabolic needs, such as de novo protein synthesis, in response to hyperosmotic shock.

Even though all the mutants exhibited the transient depolarization, three mutants (trk, hak-1 and the yeast Ptk2 homologue) were partially osmosensitive. This is consistent with roles in long-term turgor recovery: ion uptake (trk and hak-1), and activation of the $\mathrm{H}^{+}$-ATPase (Ptk2 homologue), resulting in the sustained hyperpolarization that would drive ion uptake. 
In summary, rapid changes in response to hyperosmotic shock are twofold: (1) turgor decline and hyphal shrinkage as a result of water efflux from the cell; and (2) a transient depolarization of the electrical potential. The transient depolarization results from a decline in $\mathrm{H}^{+}$-ATPase activity and increased $\mathrm{K}^{+}$permeability. Even though none of the transporters examined via knockout mutations contribute to the transient depolarization, some of them contribute to long-term turgor recovery and thus the recommencement of hyphal growth.

\section{ACKNOWLEDGEMENTS}

This research was supported in part by a discovery grant from the Natural Sciences and Engineering Research Council of Canada (R.R.L.). Special thanks to Maryam Rasti-Talkhoncheh for measuring the osmosensitivity of the transport mutants, to Vitaliy Kapishon for constructing the trk trm- 8 double mutant, and to Drs Patricia Lakin-Thomas and Sanshu Li for providing advice and assistance.

\section{REFERENCES}

Alex, L. A., Borkovich, K. A. \& Simon, M. I. (1996). Hyphal development in Neurospora crassa: involvement of a two-component histidine kinase. Proc Natl Acad Sci U S A 93, 3416-3421.

Bartnicki-Garcia, S., Bracker, C. E., Gierz, G., Lopez-Franco, R. \& Lu, H. (2000). Mapping the growth of fungal hyphae: orthogonal cell wall expansion during tip growth and the role of turgor. Biophys J 79, 2382-2390.

Beever, R. E. \& Laracy, E. P. (1986). Osmotic adjustment in the filamentous fungus Aspergillus nidulans. J Bacteriol 168, 1358-1365.

Bohnert, H. T. \& Jensen, R. G. (1996). Strategies for engineering water-stress tolerance in plants. Trends Biotechnol 14, 89-97.

Colot, H. V., Park, G., Turner, G. E., Ringelberg, C., Crew, C. M., Litvinkova, L., Weiss, R. L., Borkovich, K. A. \& Dunlap, J. C. (2006). A high through-put gene knockout procedure for Neurospora reveals functions for multiple transcription factors. Proc Natl Acad Sci U S A 103, 10352-10357.

Davis, R. H. \& de Serres, F. J. (1970). Genetic and microbiological research techniques for Neurospora crassa. Methods Enzymol 17A, 79143.

Ellis, S. W., Grindle, M. \& Lewis, D. H. (1991). Effect of osmotic stress on yield and polyol content of dicarboximide-sensitive and -resistant strains of Neurospora crassa. Mycol Res 95, 457-464.

Eraso, P., Mazon, M. J. \& Portillo, F. (2006). Yeast protein kinase Ptk2 localizes at the plasma membrane and phosphorylates in vitro the C-terminal peptide of the $\mathrm{H}^{+}$-ATPase. Biochim Biophys Acta 1758, 164-170.

Fujimura, M., Ochiai, N., Oshima, M., Motoyama, T., Ichiishi, A., Usami, R., Horikoshi, K. \& Yamaguchi, I. (2003). Putative homologs of SSK22 MAPKK kinase and PBS2 MAPK kinase of Saccharomyces cerevisiae encoded by os -4 and os-5 genes for osmotic sensitivity and fungicide resistance in Neurospora crassa. Biosci Biotechnol Biochem 67, 186-191.

Haro, R., Sainz, L., Rubio, F. \& Rodriguez-Navarro, A. (1999). Cloning of two genes encoding potassium transporters in Neurospora crassa and expression of the corresponding cDNAs in Saccharomyces cerevisiae. Mol Microbiol 31, 511-520.
Husken, D., Steudle, E. \& Zimmerman, U. (1978). Pressure probe technique for measuring water relations of cells in higher plants. Plant Physiol 61, 158-163.

Jennings, D. H. (1995). The Physiology of Fungal Nutrition, pp. 398446. Cambridge, UK: Cambridge University Press.

Jones, C. A., Greer-Philips, S. E. \& Borkovich, K. A. (2007). The response regulator RRG-1 functions upstream of a mitogen-activated protein kinase pathway impacting asexual development, female fertility, osmotic stress, and fungicide resistance in Neurospora crassa. Mol Biol Cell 18, 2123-2136.

Kaminskyj, S. G. W., Garrill, A. \& Heath, I. B. (1992). The relation between turgor and tip growth in Saprolegnia ferax: turgor is necessary, but not sufficient to explain apical extension rates. Exp Mycol 16, 64-75.

Kiranmayi, P. \& Mohan, P. M. (2006). Metal transportome of Neurospora crassa. In Silico Biol 6, 169-180.

Krantz, M., Becit, E. \& Hoffmann, S. (2006). Comparative genomics of the HOG-signaling system in fungi. Curr Genet 49, 137-151.

Lew, R. R. (1996). Pressure regulation of the electrical properties of growing Arabidopsis thaliana L. roothairs. Plant Physiol 112, 10891100.

Lew, R. R. (2005). Mass flow and pressure-driven hyphal extension in Neurospora crassa. Microbiology 151, 2685-2692.

Lew, R. R. (2007). Ionic currents and ion fluxes in Neurospora crassa hyphae. J Exp Bot 58, 3475-3481.

Lew, R. R. \& Levina, N. N. (2007). Turgor regulation in the osmosensitive cut mutant of Neurospora crassa. Microbiology 153, 1530-1537.

Lew, R. R., Levina, N. N., Walker, S. K. \& Garrill, A. (2004). Turgor regulation of hyphal organisms. Fungal Genet Biol 41, 1007-1015.

Lew, R. R., Levina, N. N., Shabala, L., Anderca, M. I. \& Shabala, S. N. (2006). Role of a mitogen-activated protein kinase cascade in ion flux-mediated turgor regulation in fungi. Eukaryot Cell 5, 480-487.

Lew, R. R., Abbas, Z., Anderca, M. I. \& Free, S. J. (2008). Phenotype of a mechanosensitive channel mutant, mid-1, in a filamentous fungus, Neurospora crassa. Eukaryot Cell 7, 647-655.

McCluskey, K. (2003). The Fungal Genetics Stock Center: from molds to molecules. Adv Appl Microbiol 52, 245-262.

Miller, T. K., Renault, S. \& Selitrennikoff, C. P. (2002). Molecular dissection of alleles of the osmotic-1 locus of Neurospora crassa. Fungal Genet Biol 35, 147-155.

Money, N. P. \& Harold, F. M. (1993). Two water molds can grow without measurable turgor pressure. Planta 190, 426-430.

Noguchi, R., Banno, S., Ichikawa, R., Fukumori, F., Ichiishi, A., Kimura, M., Yamaguchi, I. \& Fujimura, M. (2007). Identification of OS-2 MAP kinase-dependent genes induced in response to osmotic stress, antifungal agent fludioxinil, and heat shock in Neurospora crassa. Fungal Genet Biol 44, 208-218.

O'Rourke, S. M., Herskowitz, I. \& O'Shea, E. K. (2002). Yeast go the whole HOG for the hyperosmotic response. Trends Genet 18, 405412.

Philip, J. R. (1958). The osmotic cell, solute permeability, and the plant water economy. Plant Physiol 33, 264-271.

Roberts, S. K. (2003). TOK homologue in Neurospora crassa: first cloning and functional characterization of an ion channel in a filamentous fungus. Eukaryot Cell 2, 181-190.

Slayman, C. L. (1965). Electrical properties of Neurospora crassa. Effects of external cations on the intracellular potential. J Gen Physiol 49, 69-92. 
Slayman, C. L., Long, W. S. \& Lu, C. Y. (1973). The relationship between ATP and electrogenic pump in the plasma membrane of Neurospora crassa. J Membr Biol 14, 305-338.

Vogel, H. J. (1956). A convenient growth medium for Neurospora. Microb Genet Bull 13, 42-46.
Zhang, Y., Lamm, R., Pillonel, C., Lam, S. \& Xu, J.-R. (2002). Osmoregulation and fungicide resistance: The Neurospora crassa os-2 gene encodes a HOG1 mitogen-activated protein kinase homologue. Appl Environ Microbiol 68, 532-538.

Edited by: N. L. Glass 